\title{
The Effect of Light Losses in Double Integrating Spheres on Optical Properties Estimation
}

\author{
G. de Vries, J. F. Beek, G. W. Lucassen, and M. J. C. van Gemert
}

\begin{abstract}
The double integrating sphere setup (DIS) measures diffuse reflectance, diffuse transmittance and collimated transmittance, from which the optical properties of tissue (the absorption coefficient $\mu_{a}$, the scattering coefficient $\mu_{s}$ and the anisotropy of scattering $g$ ) are estimated. The effect of light losses in the DIS and optical thickness on optical properties estimation by the inverse adding doubling algorithm (IAD) and uniqueness of measurement have been investigated using a Monte Carlo method. Results were obtained for optical properties in turbid tissues $(0.80 \leq$ albedo $\leq 0.99,0.80 \leq$ anisotropy $\leq$ $0.99,1.5 \leq$ optical thickness $\leq 7.5)$ sandwiched between glass slides. At optical thickness $=6.0$ the loss of light through the glass slides is ranging from $13 \% \pm 0.5 \%$ (at albedo $=0.80$ ) to $15 \% \pm 0.5 \%$ (at albedo $=0.98)$ of the incident power. The loss of light at the exit port in the transmittance sphere is increasing upto $50 \%$ of the incident power at highly forward scattering. These losses result in a dependency on optical thickness of the optical properties estimation by the IAD algorithm. Furthermore, because of these losses, the DIS setup measurement is found to be fundamentally nonunique, when simultaneously measuring the diffuse reflectance, diffuse transmittance and collimated transmittance.
\end{abstract}

Index Terms - Absorption, anisotropy, inverse adding doubling, integrating spheres, inverse Monte Carlo, uniqueness, light losses, optical properties, scattering, tissue optics.

\section{INTRODUCTION}

$\mathbf{T}$ HE ACCURATE determination of optical properties of tissues is important for the understanding and prediction of propagation and distribution of light in tissues. In tissue optics, propagation and distribution of light in biological tissue are described by the radiative transport equation, which relates the space dependence of the radiance to the tissue optical properties. We will use the albedo $a(-)$, the optical thickness $\tau(-)$ and the anisotropy factor $g(-)$. The albedo is defined as the ratio of the scattering coefficient $\mu_{s}\left(\mathrm{~cm}^{-1}\right)$ to the attenuation coefficient $\mu_{t}=\mu_{s}+\mu_{a}$, where $\mu_{a}$ is the absorption coefficient $\left(\mathrm{cm}^{-1}\right)$. The optical thickness is defined as the sample thickness multiplied by the attenuation coefficient. The anisotropy factor is defined as the mean cosine of the scattering angle.

Up to today, many investigators have used the double integrating sphere setup (DIS) with the inverse adding dou-

Manuscript received January 4, 1999.

G. de Vries is with the Pattern Recognition Group, Delft University of Technology, 2628 CJ, Delft, The Netherlands.

J. F. Beek and M. J. C. van Gemert are with the Laser Centre, Academic Medical Center, 1105 AZ, Amsterdam ZO, The Netherlands.

G. W. Lucassen is with the Personal Care Institute, Philips Research, Eindhoven, The Netherlands.

Publisher Item Identifier S 1077-260X(99)07511-5. bling algorithm (IAD) to estimate optical properties in vitro experimentally. Measuring the diffuse reflectance $\left(R_{d}\right)$ and diffuse and collimated transmittance $\left(T_{d}\right.$ and $\left.T_{c}\right)$ from an irradiated slab of tissue held by two glass slides in between the spheres, the optical properties of the slab are estimated by solving the inverse problem. It is tacticly assumed that a unique relation exists between the three measured values of reflectance and transmittance $\left(R_{d}, T_{d}\right.$, and $\left.T_{c}\right)$ and the set of optical parameters $\left(\mu_{a}, \mu_{s}\right.$, and $\left.g\right)$ [1]-[3].

A problem may arise if light losses occurring through glass slides and ports in the spheres are not taken into account correctly in the algorithm. Beek et al. hypothesized that light losses at the sides of the sample result in overestimation of the absorption coefficient and an erroneous dependence of the obtained optical properties on the physical sample thickness [4], [5]. This especially occurs in samples with low absorption and high scattering coefficients. Monte Carlo (MC) and inverse Monte Carlo (IMC) algorithms include a correction for losses at the sides of the sample [6]-[8]. Roggan et al. avoided the effect of loss of light through the exit port in the transmittance sphere by measuring the collimated transmittance separately [7]. However, losses through the exit ports in the spheres were not reported to cause erroneous solutions.

The purpose of this paper is to show that utilization of the DIS setup to estimate optical properties of tissues fails due to: 1) light losses at the side of the sample and through the exit ports in the DIS; 2) optical thickness dependence of estimations by the IAD algorithm; and 3) nonunique DIS measurements.

\section{METHODS}

The sample with glass slides sandwiched by the two integrating spheres are considered as a "black box" with specific reflectance and transmittance. A multilayer Monte Carlo algorithm, developed by Keijzer et al. [9], has been modified to calculate the absorption in the sample, the reflectances and transmittances from the sample and loss of light through the glass slides and sphere ports. By tracing the paths of individual photons, absorption, reflectance and transmittance are estimated.

Two cases of illumination are considered: First, a collimated light beam to estimate the diffuse reflectance and diffuse transmittance at collimated irradiance, and $T_{c}$. Second, Lambertian diffuse light emitted from the sphere wall, omitting light from the apertures, to estimate the diffuse reflectance and diffuse transmittance at diffuse irradiance. The photon paths inside the sphere were not simulated. Instead the powers measured 


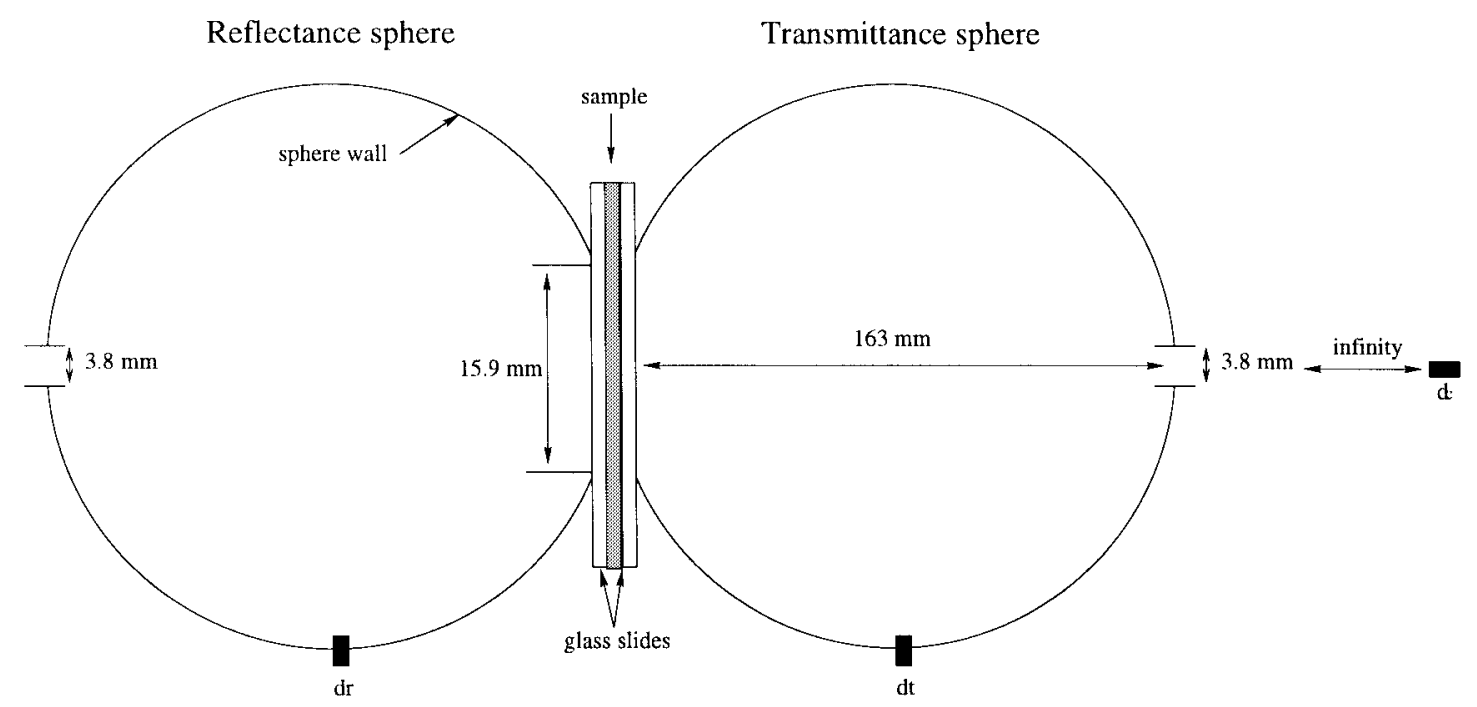

Fig. 1. The double integrating sphere setup implemented in the MC model. $\left(d_{r}, d_{t}\right.$, and $d_{c}$ are reflectance, transmittance and collimated light detectors respectively.)

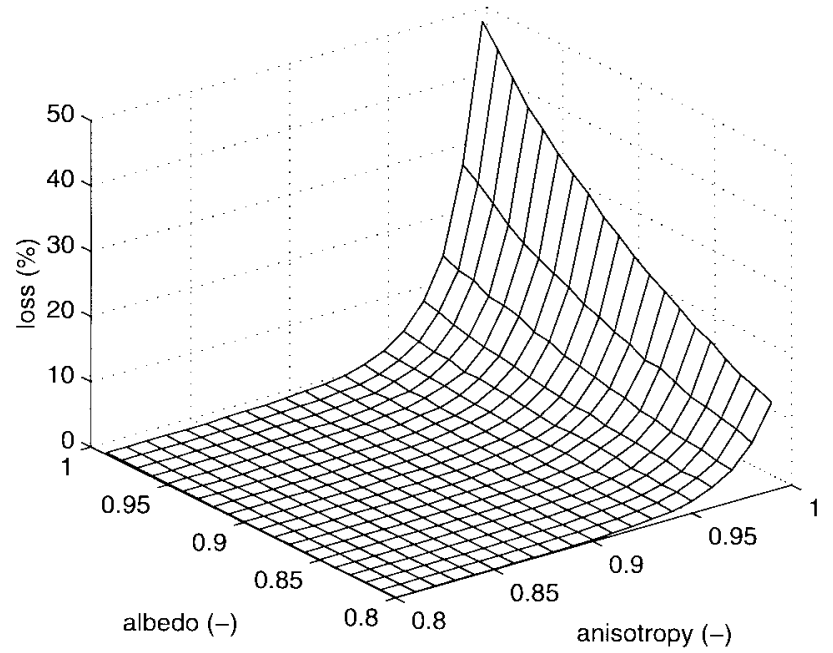

Fig. 2. Loss of light through exit port in the transmittance sphere relative to the power entering the setup. The optical thickness is $\tau=6.0$.

by detectors in the reflectance and transmittance spheres relative to the power incident to the setup were calculated analytically ( $V_{r} \%$ and $V_{t} \%$, respectively, (9) and (10) derived by Pickering et al. [1]). The collimated transmission power $\left(V_{c} \%\right)$ was calculated analytically as $\exp (\tau)$ by assuming that the probability for a scattered photon to hit the collimated transmission detector is negligible.

It is not possible to estimate the optical parameters from the measured parameter quantities analytically. Therefore, an inverse method is used. The inverse method is an iterative process of comparing relative powers estimated by means of computer simulations with measured relative powers, varying predefined optical parameters. If the results matched within the error of measurement, it was assumed that the optical parameter values were found. The minimization method used is the simplex algorithm [10].

The geometry implemented in the MC algorithm is shown in Fig. 1.

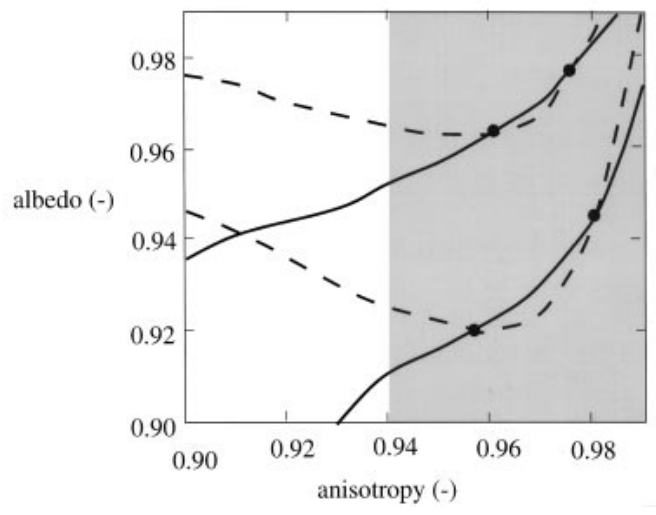

Fig. 3. Isolines of relative powers measured in the reflectance and transmittance spheres (solid: $V_{r} \%$, dashed: $V_{t} \%$, respectively) at optical thickness $\tau=6.0$. Note the dots which mark the same measured values $V \%$ at different optical parameters. The dark area represents the cases for which the IAD algorithm does not converge to a solution.

The adding-doubling (AD) algorithm calculates the reflectance and transmittance for a thin slab with predefined optical properties using the equation of radiative transfer. The reflection and transmission of a slab with arbitrary thickness are found by repeatedly doubling the thin slab until the desired thickness is reached and the reflection and transmission of a multilayer geometry are found by adding slabs. The inverse method to estimate the optical properties from DIS measurements is identical to the MC algorithm described above [3].

The effect of light losses on uniqueness of measurement and optical properties estimation was investigated at an albedo in the range $0.8 \leq a \leq 0.99$, anisotropy $0.8 \leq g \leq 0.99$ and optical thickness $\tau=6.0$ [2], [11]. The effect of optical thickness on optical properties estimation was investigated at an albedo $a=0.98$, an anisotropy $g=0.98$ and an optical thickness ranging from $1.5 \leq \tau \leq 7.5$. The refractive indices of the sample and glass slides were 1.37 and 1.5 , respectively. The thickness of the sample and glass slides was 0.5 and 0.7 $\mathrm{mm}$, respectively. 


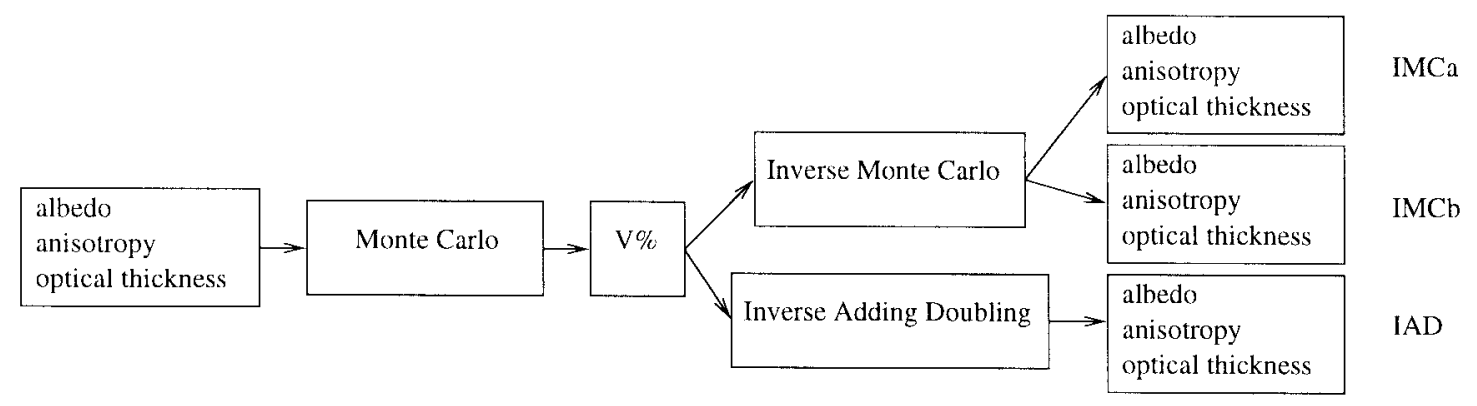

Fig. 4. Algorithm to compare IAD and IMC solutions. The input values are $a=0.98, g=0.98,1.5 \leq \tau \leq 7.5$.

\section{RESULTS}

The loss of light through the exit port in the transmittance sphere is shown in Fig. 2. The light losses through the glass slides at $0.8 \leq a \leq 0.98,0.80 \leq g \leq 0.98, \tau=6.0$ range from $13 \% \pm 0.5 \%$ to $15 \% \pm 0.5 \%$ of the incident power (from $a=$ 0.80 to $a=0.98$, respectively). The light losses through the aperture in the reflectance sphere and through the transmittance sphere for diffuse irradiation of the sample are less than $1 \%$.

Detected powers for $\tau=6.0$ are shown in Fig. 3. As a result of the increase of light loss in the transmittance sphere at high anisotropy, the relative powers measured in the transmittance sphere decrease. Hence isolines cross twice. The two detected powers $V_{r} \%$ and $V_{t} \%$ and the collimated power $V_{c} \%$ found by the IMC algorithm were used as input for the IAD algorithm. It was found that the IAD does not converge to a solution for $g>0.94$, as is depicted by the dark area in Fig. 3 .

With the MC programme the relative powers have been calculated of a sample with optical properties $a=0.98$, $g=0.98$ and varying the optical thickness $(1.5 \leq \tau \leq 7.5)$. These results have been used as input in the IMC and IAD algorithms, see Fig. 4. In Fig. 5 the results, of the procedure as described in Fig. 4, are shown of the optical properties found by the IMC and IAD algorithms. The IMC algorithm found two solutions: IMCa and IMCb.

\section{DISCUSSION}

By Prahl et al. and Yaroslavsky et al. it has been assumed that the solution of the inverse problem is unique [3], [8]. This assumption was made reasonable by plotting the total diffuse reflectance and transmittance both in the same graph. However, the light losses in the integrating sphere setup, which influence the measured powers, were not considered. The loss of light through the exit port is mainly dependent on the anisotropy factor, as is shown in Fig. 2, while the loss at the side of the sample is approximately constant. As the anisotropy and the albedo are independent optical properties, the light loss in the transmission sphere and the absorption in the sample are independent as well. Thus two absorption-loss combinations can both result in the same measured powers as is shown in Fig. 3, i.e., the DIS measurement is found to be fundamentally nonunique.

Ideally, the output of the IAD algorithm would be identical to the input of the MC algorithm if light losses do not occur. However, because of the light losses, the IAD algorithm overestimates the albedo and underestimates the anisotropy

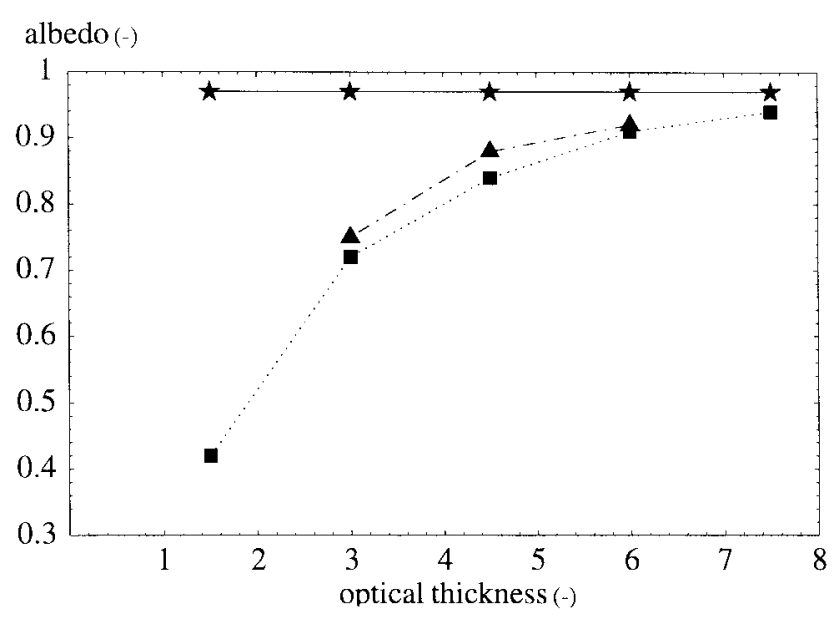

(a)

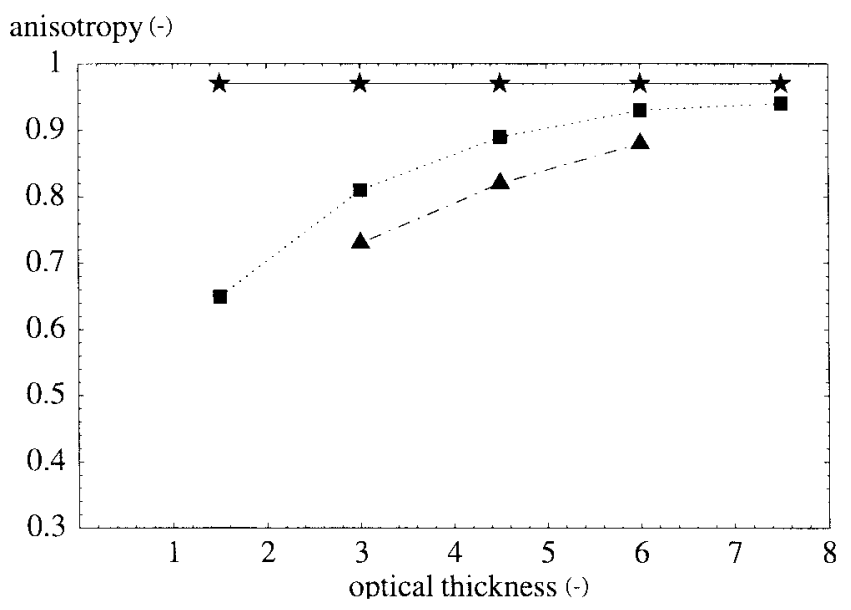

(b)

Fig. 5. (a) Albedos and (b) anisotropies found by IMC and IAD algorithms for $1.5 \leq \tau \leq 7.5$, using the algorithm shown in Fig. 4. The original input is $a=0.98, g=0.98(\star:$ IMCa. $\mathbf{m}:$ IMCb. $\mathbf{\Lambda}:$ IAD).

factor compared to the IMC algorithm. This confirms the hypothesis that DIS in combination with IAD overestimates the absorption in the sample [4], [5].

The IAD algorithm does not converge for every set of optical properties, as can be seen in Fig. 3. This has led previous investigators to discard some measurements [4]. The implicit assumption in the IAD method of negligible light loss explicitly fails in these cases. If the IMC algorithm estimates anisotropies $g>0.94$, the IAD algorithm does not converge. In case of a nonunique solution, the IAD algorithm returns 
the solution with minimal loss of light, as is shown in Fig. 5, resulting in a dependency on optical thickness.

\section{CONCLUSION}

An IMC Algorithm of the DIS setup has been developed to estimate the light losses in the DIS setup. The loss through the exit port in the transmission sphere is mainly dependent on the anisotropy of scattering. Thus, more than one albedoanisotropy combination can result in identical detected powers, i.e., the DIS setup fails to measure optical properties of highly light scattering tissues.

In case of a nonunique solution the IAD converged to the solution estimated by IMC with the highest losses. This results in a dependency of optical properties on optical thickness. If the IMC estimates $g>0.94$, the IAD algorithm does not converge. Hence, because of the light losses, the IAD algorithm fails as method to estimate optical properties from DIS measurements, in this range of anisotropy.

\section{ACKNOWLEDGMENT}

The authors wish to thank P. Frederix (Department of Physiology, Academic Medical Centre, Amsterdam, The Netherlands) and H. J. C. M. Sterenborg (Dr. Daniel den Hoed Clinic, Rotterdam, The Netherlands) for their fruitful discussions.

\section{REFERENCES}

[1] J. W. Pickering, C. J. M. Moes, H. J. C. M. Sterenborg, S. A. Prahl, and M. J. C. van Gemert, "Two integrating spheres with an intervening sample," J. Opt. Soc. Amer., vol. 9, pp. 621-631, 1993.

[2] J. W. Pickering, S. A. Prahl, N. van Wieringen, J. F. Beek, and M. J. C. van Gemert, "Double integrating sphere system for measuring optical properties of tissue," Appl. Opt., vol. 32, pp. 399-410, 1993.

[3] S. A. Prahl, M. J. C. van Gemert, and A. J. Welch, "Determining the optical properties of turbid media by using the adding-doubling method," Appl. Opt., vol. 32, pp. 559-568, 1993.

[4] J. F. Beek, H. J. van Staveren, P. Posthumus, H. J. C. M. Sterenborg, and M. J. C. van Gemert, "The optical properties of lung as a function of respiration," Phys. Med. Biol., vol. 42, pp. 2263-2272, 1997.

[5] J. F. Beek, P. Blokland, P. Posthumus, M. Aalders, J. W. Pickering, H. J. C. M. Sterenborg, and M. J. C. van Gemert, "In vitro doubleintegrating-sphere optical properties of tissues between 630 and 1064 nm," Phys. Med. Biol., vol. 42, pp. 2255-2261, 1997.

[6] C. J. Hourdakis and A. Perris, "Monte Carlo estimation of tissue optical properties for use in laser dosimetry," Phys. Med. Biol., vol. 40, pp. 351-364, 1995

[7] A. Roggan, H. Albrecht, K. Dorschel, O. Minet, and G. Muller, "Experimental set up and Monte Carlo model for determination of optical tissue properties in the wavelength range 330-1100 nm," Proc. SPIE, Laser Interaction with Hard and Soft Tissue II, 1994, vol. 2323, pp. 1-16.

[8] V. Yaroslavsky and V. V. Tuchin, "An Inverse Monte Carlo method for spectrophotometric data processing," Proc. SPIE, Cell and Biotissue Optics, vol. 2100, pp. 57-66, 1994.

[9] M. Keijzer, S. L. Jacques, S. A. Prahl, and A. J. Welch, "Light distributions in artery tissue: Monte Carlo simulations for finite laser beams," Lasers Surg. Med., vol. 9, pp. 148-154, 1989.
[10] W. H. Press, B. P. Flannery, P. S. A. Teukolsky, and W. T. Vetterling, Numerical Recipes in Pascal. Cambridge, U.K.: Cambridge Univ. Press, 1989.

[11] W. F. Cheong, S. A. Prahl, and A. J. Welch, "Review of optical properties of biological tissues," IEEE J. Quantum Electron., vol. 25 , pp. 2166-2185, 1990.

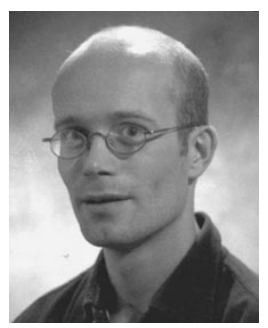

G. de Vries received the Masters degree in physics at the University of Amsterdam, The Netherlands, in 1996. Since 1997, he is working toward the Ph.D. degree at the Pattern Recognition Group, Delft University of Technology, Delft, The Netherlands.

$\mathrm{He}$ has been working at the Laser Center of the Academic Medical Center since 1994.

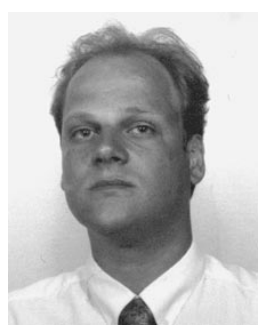

J. F. Beek received the medical degree in 1998. He has been working in medical laser applications since 1987, completed his $\mathrm{PhD}$ thesis in 1993.

Since then, he has been a senior-investigator at the Laser Center of the Academic Medical Center in Amsterdam, The Netherlands.

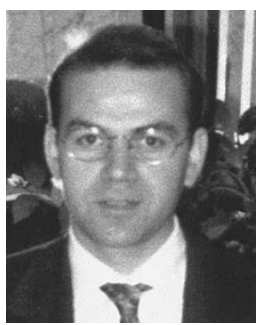

G. W. Lucassen studied physics at the Technical University Twente, The Netherlands, worked for the EARS company in Delft, at the Laser Center of the Academic Medical Center in Amsterdam.

$\mathrm{He}$ is currently working for Philips at their Research Laboratories in Eindhoven, The Netherlands.

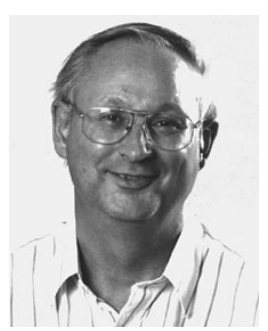

M. J. C. van Gemert studied physics at Delft University of Technology.

He worked at the Philips Research Laboratories and the St. Joseph Hospital, both in Eindhoven. Since 1987, he is director of the Laser Centre of the Academic Medical Center in Amsterdam, The Netherlands, and since 1990, a Professor in Medical Applications of Laser Physics. 